\title{
Uso de hidrocolóide e alginato de cálcio no tratamento de lesões cutâneas
}

\author{
Use of hydrocolloid and calcium alginate in the treatment of skin lesions
}

Uso de hidrocoloide y alginato de calcio en el tratamiento de lesiones de la piel

\author{
Luciane da Silva Pinheiro', Eline Lima Borges', Miguir Terezinha Vieccelli Donoso' \\ ' Universidade Federal de Minas Gerais, Escola de Enfermagem, \\ Departamento de Enfermagem Básica. Belo Horizonte-MG, Brasil.
}

Submissão: 06-07-2011 Aprovação: 27-08-2013

\section{RESUMO}

Hidrocolóide e alginato de cálcio são utilizados no tratamento de lesões cutâneas e muitos profissionais da saúde desconhecem as evidências que sustentam sua indicação na cicatrização. Objetivou-se nesse estudo identificar evidências da ação da placa de hidrocolóide e alginato de cálcio no tratamento de lesão cutânea. Utilizou-se o método de revisão integrativa da literatura. Fizeram parte da amostra 12 estudos com nível de evidências I, II, III e IV, formados por pacientes com lesão cutânea tratada durante qualquer período de tempo com hidrocolóide placa ou alginato de cálcio. Os desfechos avaliados foram redução da área, cicatrização da lesão, taxa de cicatrização, tempo e infecção. Os resultados permitiram estabelecer três recomendações para o uso de hidrocolóide e nenhuma para uso de alginato de cálcio no tratamento de lesão crônica.

Descritores: Curativos Hidrocolóides; Alginato; Curativos Oclusivos; Cicatrização de Lesões.

ABSTRACT
Hydrocolloid and calcium alginate are used to treat cutaneous injuries and many health professionals do not know about its cicatrization effects. This study had the objective to identify the evidences of the action of the hydrocolloid and calcium alginate dressing in the treatment of cutaneous injuries. The method of integrative review was used. 12 studies with evidence levels I, II, III and IV were part of the sample; they were composed by patients with cutaneous injuries treated in any period of time with hydrocolloid dressing or calcium alginate. The evaluated outcomes had been reduction of the area, cicatrization of the injury, rate of cicatrization, time and infection. The results had allowed establishing three recommendations for the use of hydrocolloid and none for use of alginate of calcium in the treatment of chronic injury.

Key words: Bandages Hydrocolloid; Alginates; Occlusive Dressings; Injury Cicatrizatio.

\section{RESUMEN}

El hidrocoloide y el alginato de calcio se utilizan en el tratamiento de lesiones en la piel y muchos profesionales de la salud desconocen sus efectos sobre la cicatrización de heridas. Este estudio pretende identificar evidencia de acción de placas de alginato de calcio y de hidrocoloide para el tratamiento de la lesión cutánea. Se utilizó el método de revisión integradora. Fueran incluidos en la muestra 12 estudios con nivel de evidencia I, II, III y IV, formado por pacientes con lesión cutánea tratado durante cualquier período de tiempo con hidrocoloide placa o con alginato de calcio. Los resultados evaluados fueran reducción del área, cicatrización de lesión, tasa de cicatrización, tiempo e infección. Los resultados han permitido establecer tres recomendaciones para el uso del hidrocoloide y ninguna para el uso del alginato de calcio en el tratamiento de heridas. Palabras clave: Vendas Hidrocoloidales; Alginates; Apósitos Oclusivos; Cicatrización de Lesiones.

\section{AUTOR CORRESPONDENTEＥline Lima Borges E-mail: eborges@ufmg.br}




\section{INTRODUÇÃO}

O conceito de cicatrização úmida de lesão tem sido examinado e gradualmente aceito por profissionais de saúde que cuidam de pessoas com lesão cutânea durante os últimos 40 anos e tem levado ao desenvolvimento de centenas de coberturas que oferecem suporte para a manutenção de ambiente úmido na lesão(1).

Coberturas são elaboradas para manter a lesão limpa e livre de contaminação e também para promover a cicatrização, principalmente de lesões crônicas, que podem apresentar perda significativa de tecido ${ }^{(2)}$.

Para a obtenção de um ambiente adequado à cicatrização de lesões, recomenda-se o uso de coberturas oclusivas capazes de reduzir a tensão de $\mathrm{O}_{2}$ na superfície lesada. Elas também devem ser interativas, de superfície impermeáveis ou semipermeáveis à troca gasosa, garantindo uma umidade fisiológica no leito da lesão, o que evita a formação de crosta ou maceração. A interatividade exige que a cobertura tenha propriedade de manter a temperatura da lesão em torno de $37^{\circ} \mathrm{C}$, estimulando a mitose celular. Hidrocolóides e alginato são alguns exemplos de coberturas oclusivas disponíveis no mercado.

No Brasil, muitos profissionais desconhecem a indicação das coberturas de hidrocolóide e alginato de cálcio, classificadas como interativas. A sua ação na cura das lesões ainda não está suficientemente estabelecida na prática clínica. Na maioria das vezes os profissionais trabalham sem amparo em evidências científicas. Embora o hidrocolóide e o alginato de cálcio sejam coberturas frequentemente utilizadas no tratamento de lesões agudas e crônicas, ainda persistem dúvidas a respeito de suas indicações. O seu uso ainda não se baseia em evidências científicas, o que interfere de forma negativa no processo de padronização dos produtos em muitos serviços de saúde.

A identificação de evidências para a indicação da cobertura de hidrocolóide e alginato de cálcio se justifica, pois esse conhecimento irá amparar os profissionais na escolha e indicações correta desses produtos. Espera-se também contribuir com os serviços de saúde na elaboração de protocolos de atendimento a pacientes com lesões, subsidiados por evidências científicas.

\section{OBJETIVO}

Identificar as evidências para indicação da placa de hidrocolóide e do alginato de cálcio no tratamento de lesão cutânea.

\section{MÉTODO}

Para o desenvolvimento do estudo utilizou-se a revisão integrativa de literatura. O método de revisão integrativa é uma abordagem que permite a inclusão de metodologias diversificadas (investigação experimental e não experimental) e tem o potencial de desempenhar um papel maior na prática baseada em evidências, pois permite a compreensão do fenômeno analisado. Além disso, contribui para a apresentação de diversas perspectivas sobre um fenômeno de preocupação e tem sido defendida como importante para a ciência e a prática da enfermagem ${ }^{(3)}$.

Para essa revisão foram percorridas seis etapas, recomendadas pela literatura: elaboração da pergunta norteadora; busca ou amostragem na literatura; coleta de dados; análise crítica dos estudos incluídos; discussão dos resultados e apresentação da revisão integrativa. A revisão deve ser clara e completa para que o leitor avalie criticamente os resultados, que devem conter informações pertinentes e detalhadas com base em metodologias contextualizadas, sem omissão de qualquer evidência relacionada ${ }^{(3)}$.

Inicialmente, formulou-se a seguinte questão: quais são as ações da placa de hidrocolóide e do alginato de cálcio no tratamento de lesão cutânea?

A coleta de dados ocorreu no período de junho a dezembro de 2009, e foi atualizada no período de 01/07/13 a 29/07/13, nas seguintes bases de dados: Biblioteca Cochrane (COCHRANE), Medical Literature Analysis and Retrieval Sistem on-line (MEDLINE) e Literatura Latino-Americana e do Caribe e em Ciências da Saúde (LILACS).

Para a identificação dos artigos nas bases de dados foram utilizados os descritores controlados Hydrogel; Colloids; Alginates; Bandages; Bandages, Hydrocolloid; Occlusive Dressings; Varicose Ulcer; Pressure Ulcer; Granulation Tissue; Skin Ulcer; Wounds and Injuries; Wound Healing e Ulcer.

A composição da amostra respeitou os critérios de inclusão: artigos publicados em português, inglês e espanhol no período de 2003 a 2013, de estudos de metanálise ou estudos clínicos randomizados controlados ou estudos clínicos não randomizados controlados ou estudos descritivos, classificados em nível de evidência I, II, III e IV, respectivamente ${ }^{(4)}$.

Para que fossem selecionados, os artigos deveriam ter por amostra pacientes com lesão cutânea aguda ou crônica, independentemente da etiologia, submetida a tratamento com placa de hidrocolóide ou alginato de cálcio durante qualquer período de tempo e o resultado avaliado (desfecho) referir-se a redução da área lesada ou cicatrização da lesão ou taxa de cicatrização ou tempo de cicatrização ou infecção.

Para a coleta de dados foi elaborado um instrumento composto por dados referentes ao periódico (nome, ano, volume, número, idioma), à autoria (número de autores, nome do autor principal) e ao estudo (local da pesquisa, identificação da amostra, desenho, tipo de participantes, etiologia da lesão, tipo de intervenções, resultados, conclusão e nível de evidência do estudo).

A estratégia de busca nas bases de dados, número de estudos identificados, selecionados e os que compuseram a amostra estão descritos no Quadro 1.

$\mathrm{Na}$ base dados Cochrane foram identificados 31 artigos. $\mathrm{Na}$ base dados Medline utilizou-se duas estratégias de busca. $\mathrm{Na}$ primeira deu-se enfoque para publicações de estudos de metanálise, obtendo-se 24 artigos e selecionados quatro. Na segunda estratégia ampliou-se tipo de desenho das publicações, identificando-se 158 artigos, dos quais foram selecionados 11, porém cinco artigos já haviam sido identificados na base Cochrane e quatro na primeira estratégia de busca no Medline, não sendo aceitos para comporem a amostra. Na base LILACS foram identificadas 44 publicações e selecionada uma. 
Quadro 1 - Estratégia de busca, estudos identificados e selecionados. Belo Horizonte-MG, 2013.

\begin{tabular}{|c|c|c|c|c|}
\hline \multirow{2}{*}{ Base de Dados } & \multirow{2}{*}{ Estratégia de Busca } & \multicolumn{3}{|c|}{ Estudos } \\
\hline & & Identificados & Selecionados & Da amostra \\
\hline COCHRANE & $\begin{array}{l}\text { Alginates OR Hydrogel OR Bandagens, Hydrocolloid } \\
\text { AND Wounds and injuries }\end{array}$ & 31 & 05 & 05 \\
\hline \multirow[b]{2}{*}{ MEDLINE } & 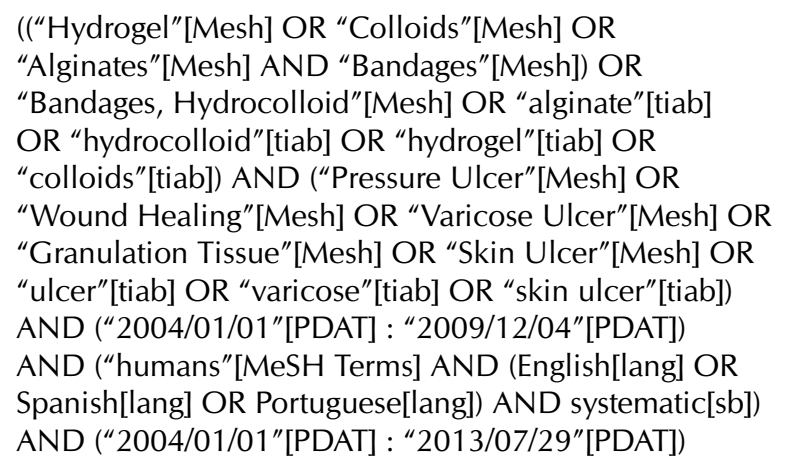 & 24 & 04 & 04 \\
\hline & 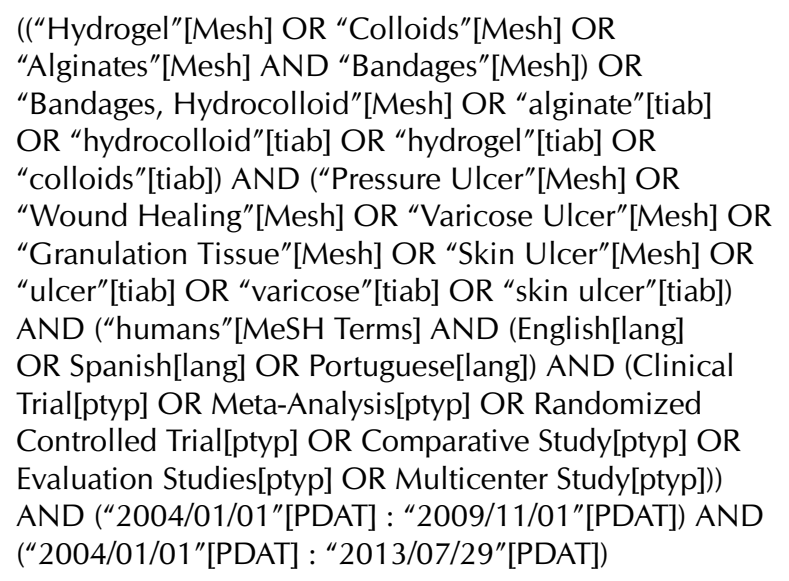 & 158 & 11 & 02 \\
\hline LILACS & 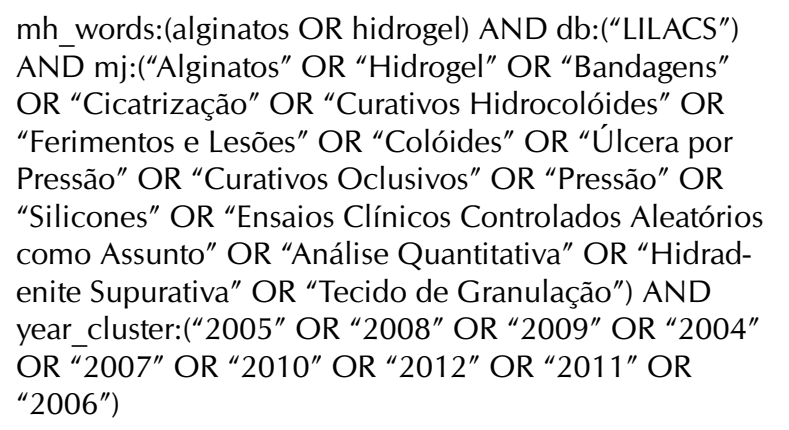 & 44 & 01 & 01 \\
\hline TOTAL & & 257 & 21 & 12 \\
\hline
\end{tabular}

Legenda: Cinco artigos já haviam sido identificados na base Cochrane e quatro na primeira estratégia de busca no Medline

Os 257 artigos identificados foram submetidos à leitura do título e resumo, sendo que, ao final dessa etapa, foram selecionados 21. Desses, 12 atendiam aos critérios de inclusão e compuseram a amostra, sendo nove sobre placa de hidrocolóide, dois sobre alginato de cálcio e um sobre ambos.

\section{RESULTADOS}

Para fins didáticos, os artigos foram codificados de A a L. Os dados referentes ao periódico e autoria estão apresentados no Quadro2. 
O período de publicação dos artigos variou de 2004 a 2008. Destaca-se que não foram identificados estudos publicados nos anos de 2006 e no período de 2009 a 2013 nas bases pesquisadas. No ano de 2004 foram publicados três estudos e, o maior número de publicações (quatro) ocorreu no ano de 2008, sendo todos sobre hidrocolóide. Nos anos de 2004 e 2007 foram obtidos três estudos em cada ano.
Todos os estudos foram publicados em periódicos internacionais, sendo dois da Ásia, um da América Latina e nove da Europa. O idioma inglês foi utilizado em 11 artigos e o espanhol em um. A maioria (08) dos estudos foi realizada por mais de três autores, três por três autores e dois tinham uma autoria.

No Quadro 3 encontram-se a síntese do objetivo, método e resultados dos estudos da amostra.

Quadro 2 - Apresentação dos dados referentes ao periódico e autoria dos estudos da amostra. Belo Horizonte-MG, 2013.

\begin{tabular}{|c|c|c|c|c|}
\hline Codificação & Periódico & $\begin{array}{l}\text { Data de } \\
\text { publicação }\end{array}$ & Título do artigo & Nome dos autores \\
\hline Estudo $A^{(5)}$ & Asian / Surg; & 2004 & $\begin{array}{l}\text { Meta-analysis of randomized controlled trials } \\
\text { on hydrocolloid occlusive dressing versus } \\
\text { conventional gauze dressing in the healing of } \\
\text { chronic wounds. }\end{array}$ & $\begin{array}{l}\text { Singh A, Halder S, } \\
\text { Menon GR, Chumber S, } \\
\text { Misra MC, Sharma LK, } \\
\text { Srivastava A. }\end{array}$ \\
\hline Estudo $B^{(6)}$ & BMJ on line. & 2007 & $\begin{array}{l}\text { Dressings for venous leg ulcers: systematic } \\
\text { review and meta-analysis }\end{array}$ & $\begin{array}{l}\text { Palfreyman S, Nelson A, } \\
\text { Jonathan A, Michaels JA. }\end{array}$ \\
\hline Estudo $C^{(7)}$ & I Vasc Surg. & 2007 & $\begin{array}{l}\text { A factorial, randomized trial of pentoxifylline } \\
\text { or placebo, four-layer or single-layer } \\
\text { compression, and knitted viscose or } \\
\text { hydrocolloid dressings for venous ulcers. }\end{array}$ & $\begin{array}{l}\text { Nelson EA, Prescott RJ, } \\
\text { Harper DR, Gibson B, } \\
\text { Brown D, Ruckley CV. }\end{array}$ \\
\hline Estudo $\mathrm{D}^{(8)}$ & BMC Dermatol. & 2004 & $\begin{array}{l}\text { A randomized clinical trial comparing } \\
\text { hydrocolloid, phenytoin and simple dressings } \\
\text { for the treatment of pressure ulcers }\end{array}$ & $\begin{array}{l}\text { Hollisaz MT, Khedmat } \\
\text { H, Yari F. }\end{array}$ \\
\hline Estudo $\mathrm{E}^{(9)}$ & $\begin{array}{l}\text { Adv Skin Wound } \\
\text { Care }\end{array}$ & 2008 & $\begin{array}{l}\text { A prospective, randomized, multisite clinical } \\
\text { evaluation of a transparent absorbent acrylic } \\
\text { dressing and a hydrocolloid dressing in the } \\
\text { management of Stage II and shallow Stage III } \\
\text { pressure ulcers. }\end{array}$ & $\begin{array}{l}\text { Brown-Etris M, Milne } \\
\text { C, Orsted H, Gates JL, } \\
\text { Netsch D, Punchello M, } \\
\text { Couture N, Albert M, } \\
\text { Attrell E, Freyberg J. }\end{array}$ \\
\hline Estudo $F^{(10)}$ & Burns & 2005 & $\begin{array}{l}\text { Biobrane }{ }^{\circledR} \text { versus Duoderm }{ }^{\circledR} \text { for the treatment } \\
\text { of intermediate thickness burns in children: a } \\
\text { prospective, randomized trial. }\end{array}$ & Cassidy C. \\
\hline Estudo $G^{(11)}$ & Asian / Surg & 2008 & $\begin{array}{l}\text { Prospective evaluation of occlusive } \\
\text { hydrocolloid dressing versus conventional } \\
\text { gauze dressing regarding the healing effect } \\
\text { after abdominal operations: Randomized } \\
\text { controlled trial. }\end{array}$ & $\begin{array}{l}\text { Shinohara T, Yamashita } \\
\text { Y, Satoh K, Mikami K, } \\
\text { Yamauchi Y, Hoshino S, } \\
\text { Noritomi A, Maekawa T. }\end{array}$ \\
\hline Estudo $\mathrm{H}^{(12)}$ & Surg Neurol. & 2008 & $\begin{array}{l}\text { Clinical evaluation of hydrocolloid dressings } \\
\text { for neurosurgical wounds. }\end{array}$ & $\begin{array}{l}\text { Fujimoto Y, Shimooka N, } \\
\text { Ohmishi Y, Yoshimine T. }\end{array}$ \\
\hline Estudo I I3) & Rev. Méd. Urug. & 2008 & $\begin{array}{l}\text { Apósitos hidrocoloidales en la cicatrización } \\
\text { por segunda intención de heridas quirúrgicas. }\end{array}$ & $\begin{array}{l}\text { Bazzano C, Alvarez M, } \\
\text { Martínez M. }\end{array}$ \\
\hline Estudo J J14) & Rev Enferm. & 2005 & $\begin{array}{l}\text { Therapeutic behavior of a hydrocolloid } \\
\text { dressing. Its evolution in the treatment of } \\
\text { acute and chronic dermal ulcers. }\end{array}$ & $\begin{array}{l}\text { Aparicio EG, Castilla } \\
\text { PCC, Díez GMT, Dorado } \\
\text { A, Mendoza G, Núñez } \\
\text { FJM, Pascual RJ. }\end{array}$ \\
\hline Estudo $K^{(15)}$ & Diabet Med & 2007 & $\begin{array}{l}\text { Prospective randomized controlled study of } \\
\text { Hydrofiber dressing containing ionic silver or } \\
\text { calcium alginate dressings in non-ischaemic } \\
\text { diabetic foot ulcers. }\end{array}$ & $\begin{array}{l}\text { Jude EB, Apelqvist J, } \\
\text { Spraul M, Martini J. }\end{array}$ \\
\hline Estudo $L^{(16)}$ & $\begin{array}{l}\text { British Journal of } \\
\text { Nursing. }\end{array}$ & 2004 & $\begin{array}{l}\text { Comparison of four different dressings on } \\
\text { donor site wounds. }\end{array}$ & Beldon P. \\
\hline
\end{tabular}




\begin{tabular}{|c|c|c|c|c|}
\hline 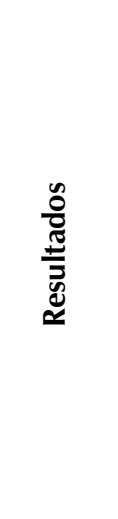 & 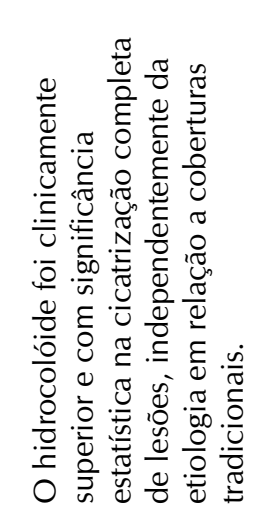 & 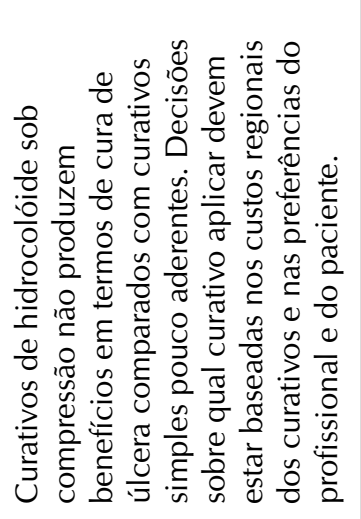 & 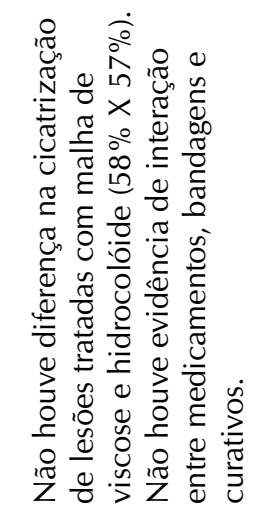 & 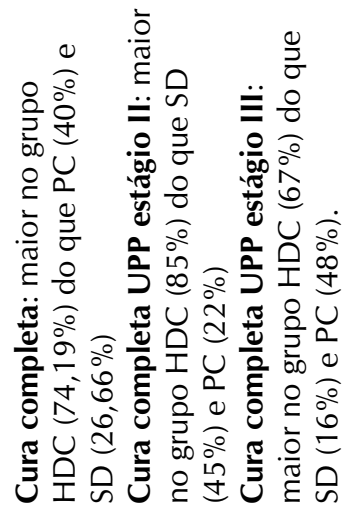 \\
\hline 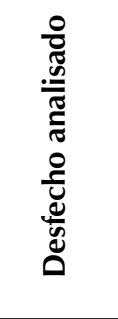 & 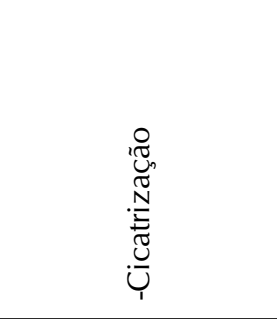 & 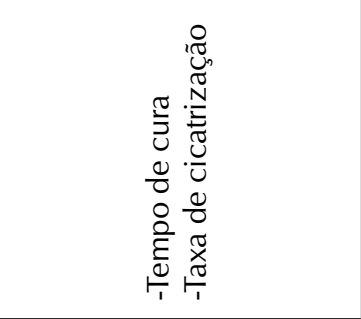 & 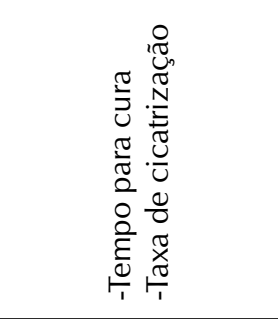 & 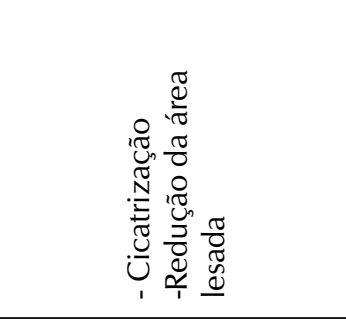 \\
\hline 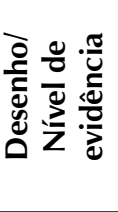 & 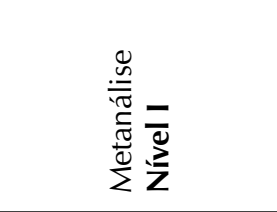 & 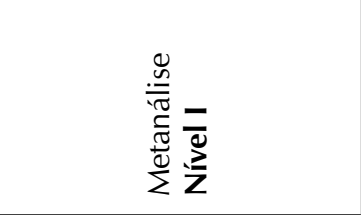 & 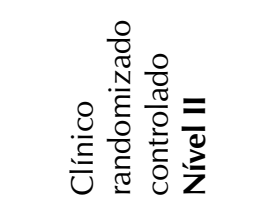 & 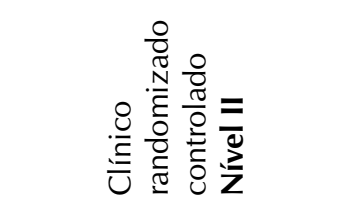 \\
\hline 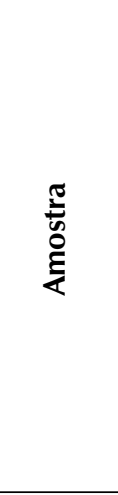 & 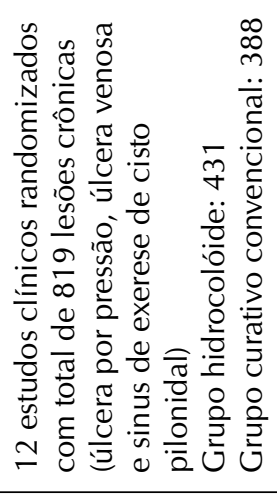 & 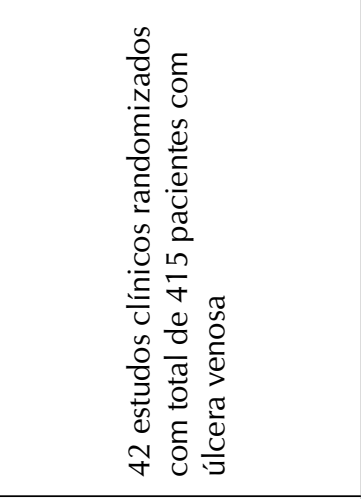 & 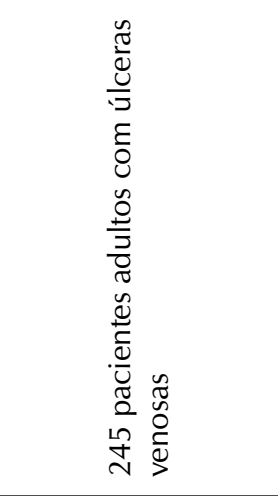 & 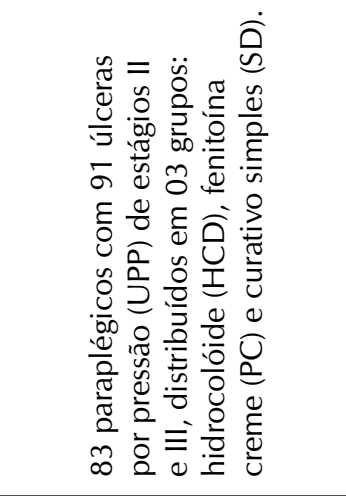 \\
\hline$\frac{\stackrel{0}{\frac{1}{0}}}{\frac{0}{0}}$ & 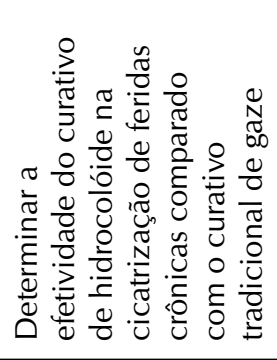 & 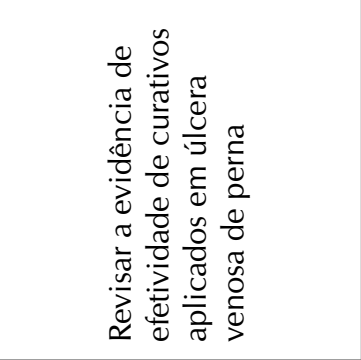 & 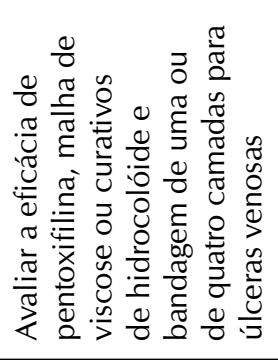 & 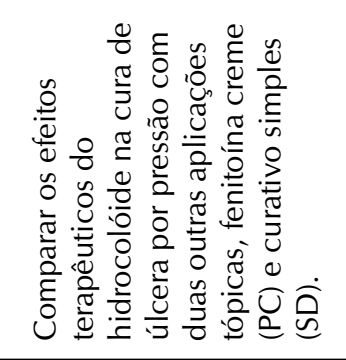 \\
\hline 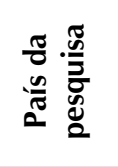 & $\begin{array}{l}\stackrel{\tilde{\sigma}}{\bar{\sigma}} \\
\underline{\underline{\Xi}}\end{array}$ & 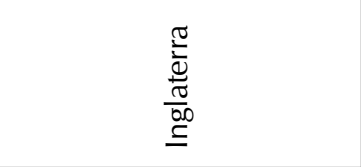 & 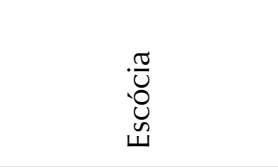 & $\stackrel{\mathscr{T}}{=}$ \\
\hline 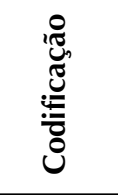 & $\begin{array}{l}\frac{\sqrt{0}}{4} \\
\frac{0}{0} \\
\sum_{0}^{2} \\
\text { w }\end{array}$ & 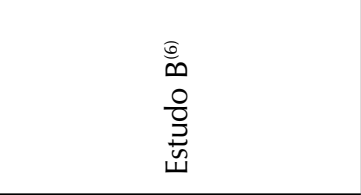 & 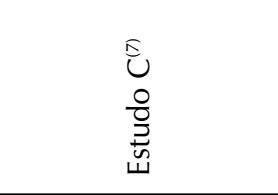 & 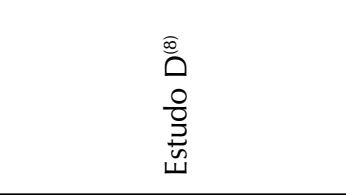 \\
\hline
\end{tabular}




\begin{tabular}{|c|c|c|c|c|}
\hline 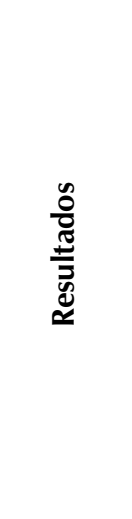 & 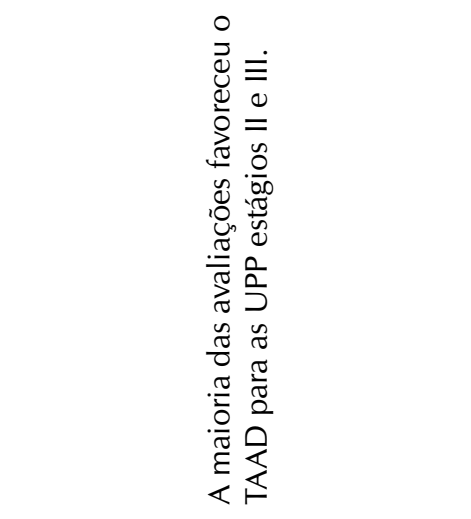 & 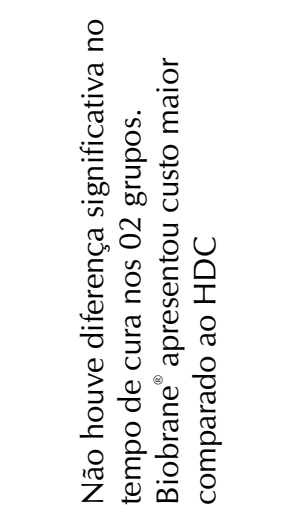 & 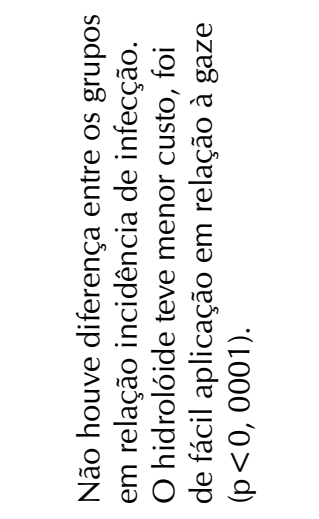 & 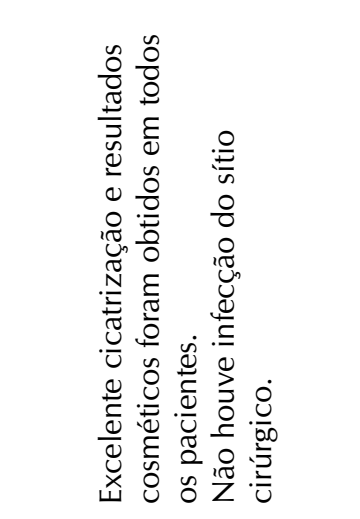 \\
\hline 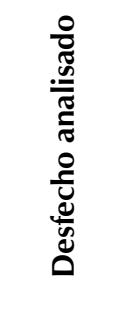 & 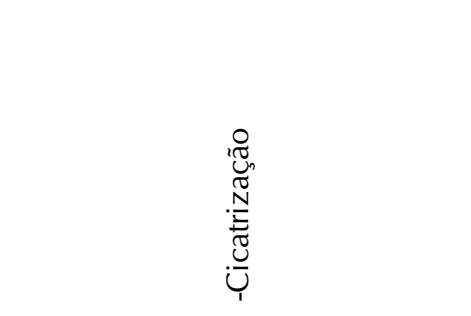 & 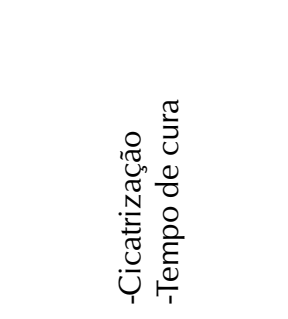 & 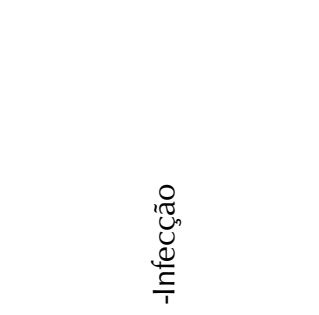 & 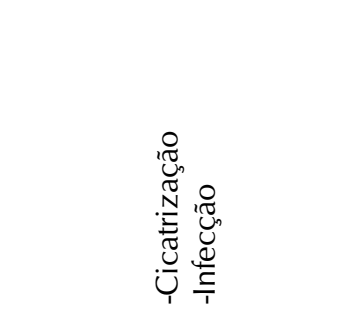 \\
\hline 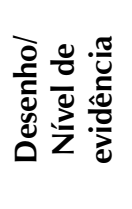 & 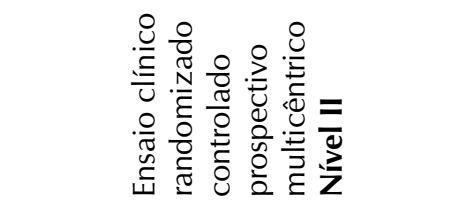 & 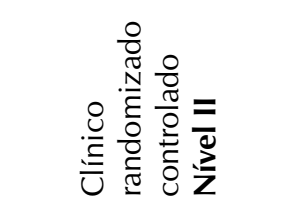 & 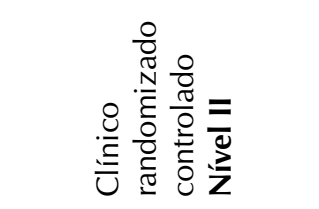 & 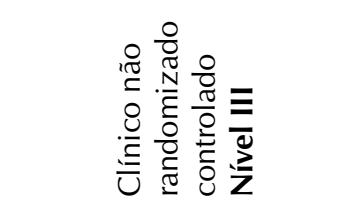 \\
\hline$\frac{5}{\grave{5}}$ & 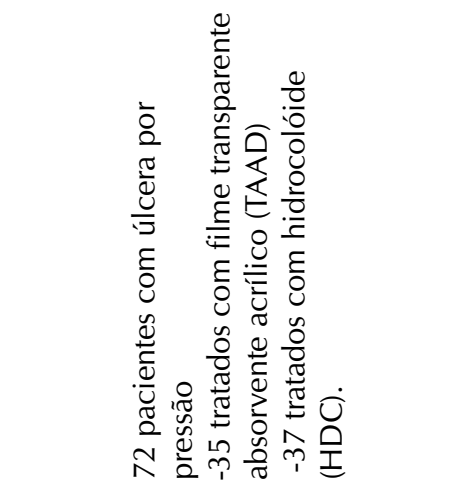 & 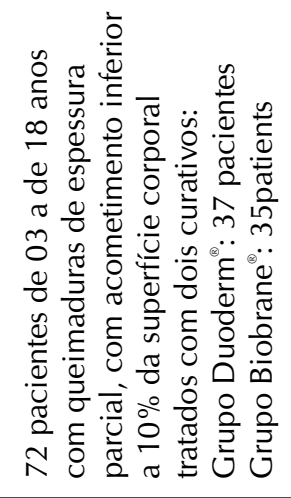 & 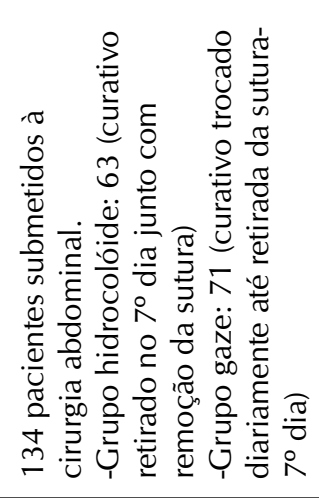 & 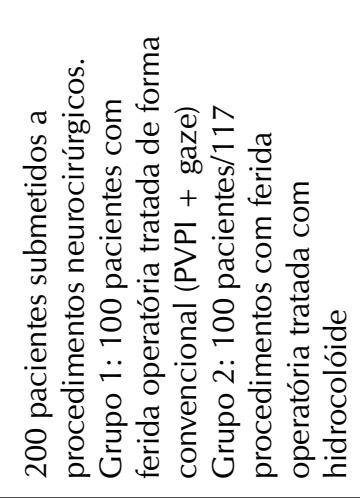 \\
\hline 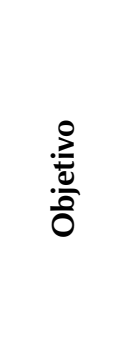 & 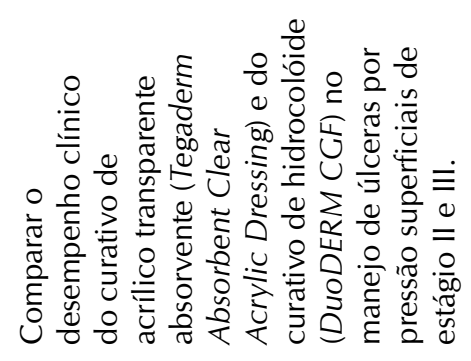 & 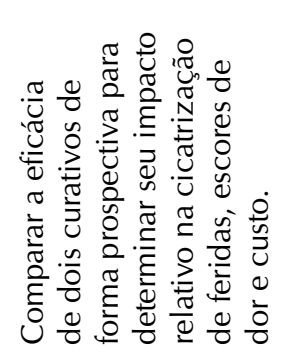 & 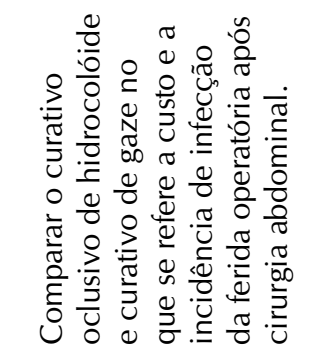 & 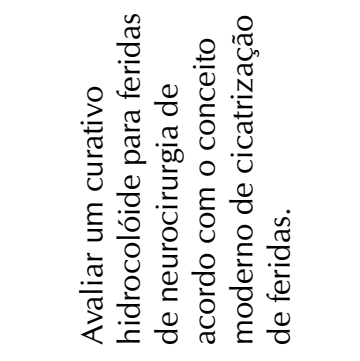 \\
\hline 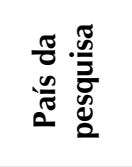 & 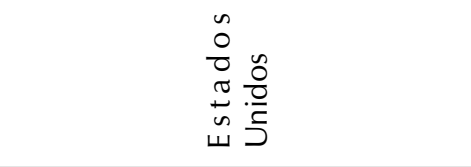 & 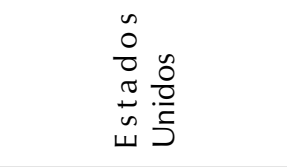 & 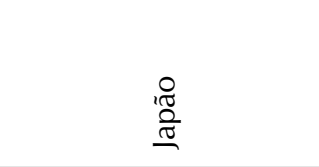 & $\begin{array}{l}\stackrel{\mathscr{\pi}}{0} \\
\frac{\pi}{\pi}\end{array}$ \\
\hline : & 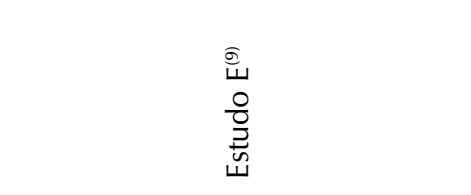 & 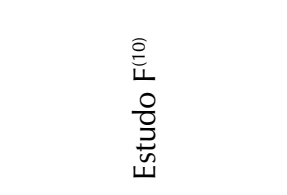 & $\begin{array}{l}\text { 总 } \\
\text { O } \\
\text { 을 } \\
\text { 总 }\end{array}$ & 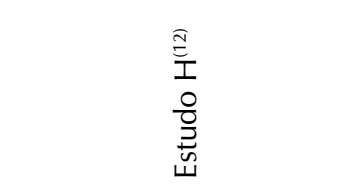 \\
\hline
\end{tabular}




\begin{tabular}{|c|c|c|c|c|}
\hline 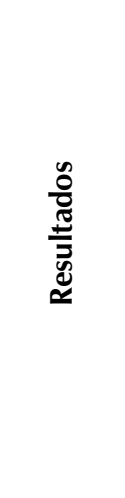 & 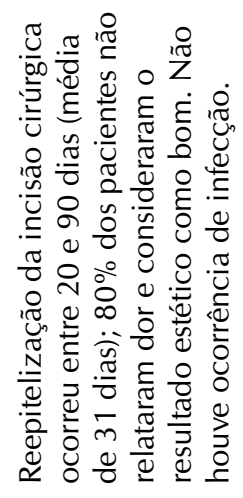 & 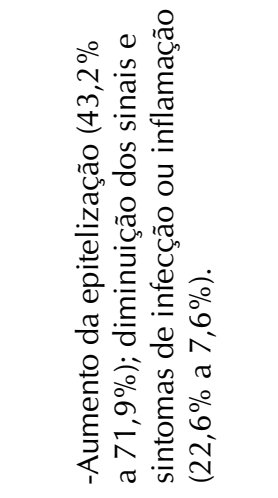 & 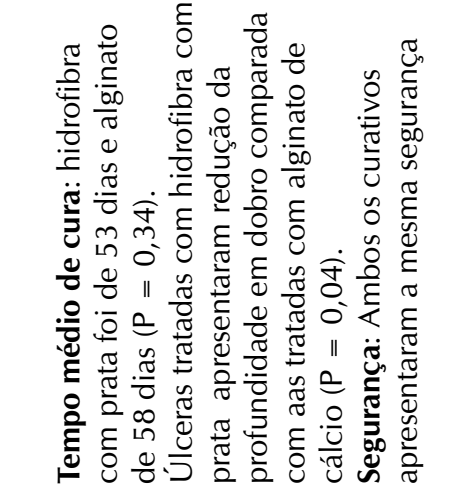 & 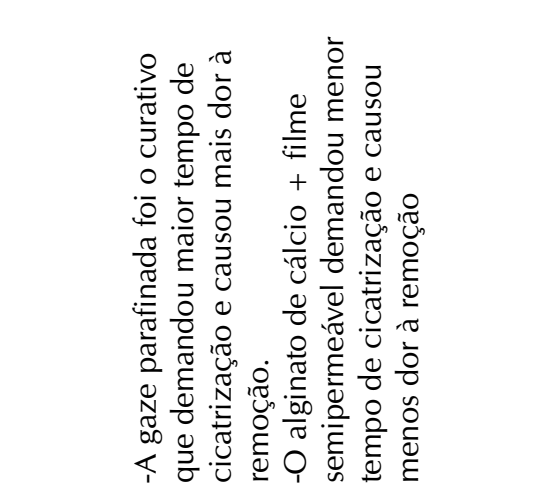 \\
\hline 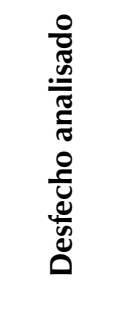 & 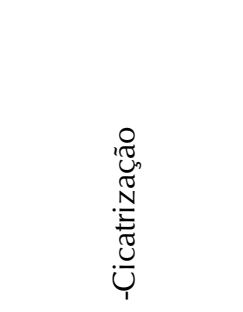 & 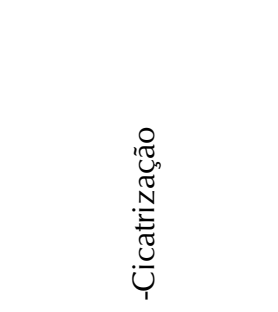 & 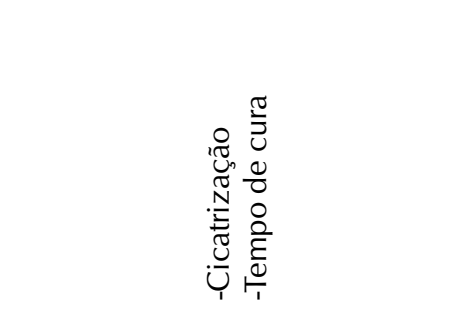 & 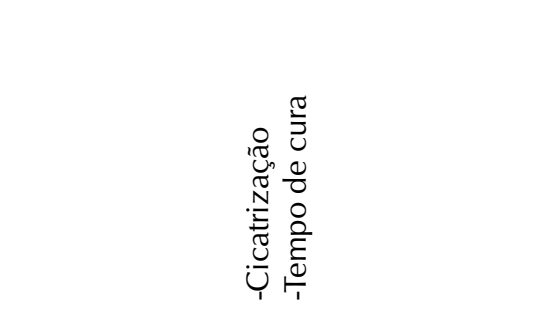 \\
\hline 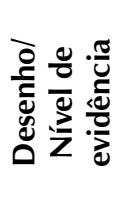 & 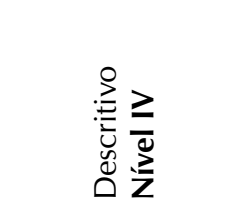 & 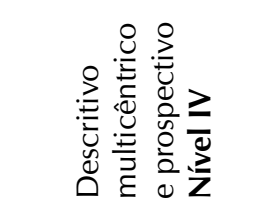 & 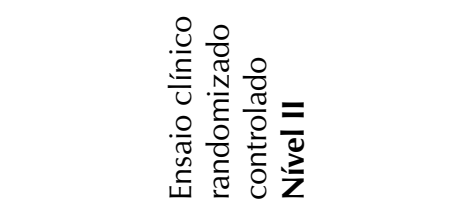 & 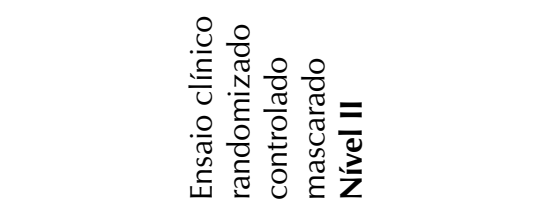 \\
\hline 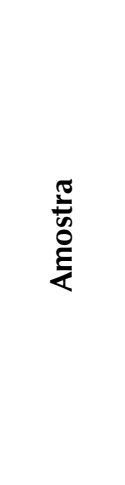 & 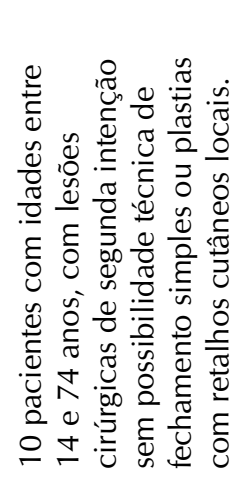 & 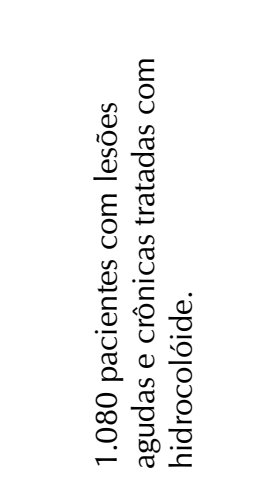 & 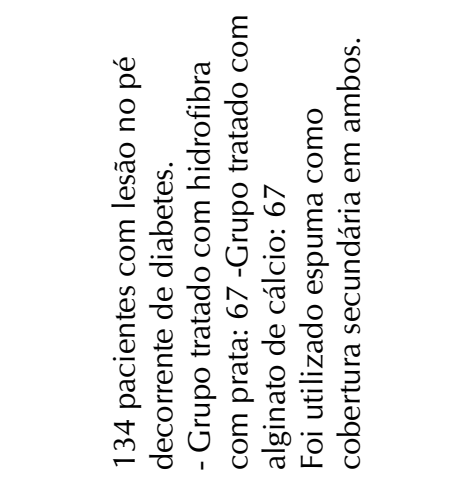 & 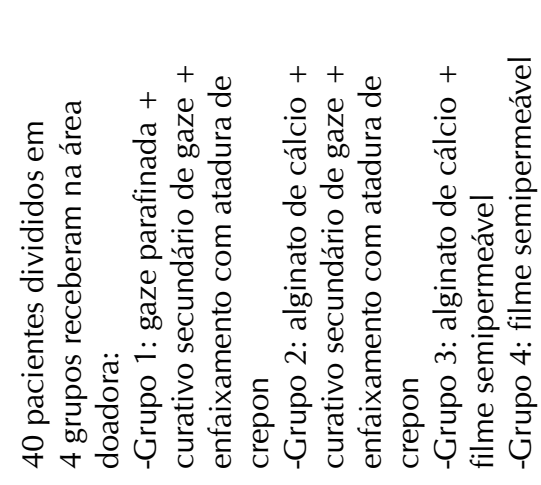 \\
\hline$\frac{\stackrel{0}{0}}{\frac{0}{0}}$ & 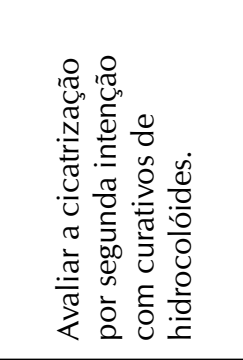 & 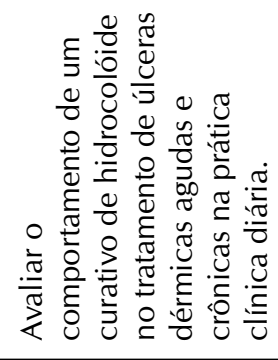 & 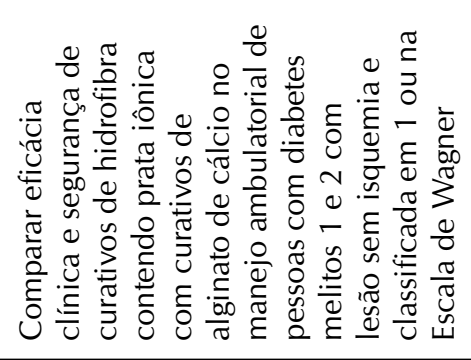 & 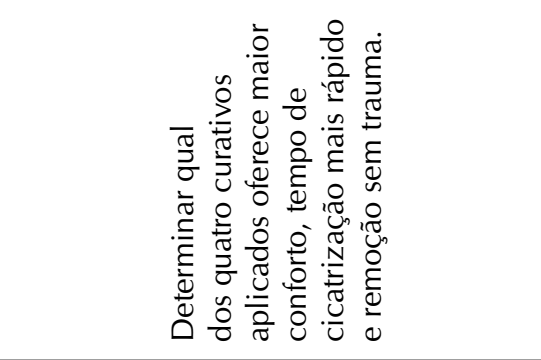 \\
\hline 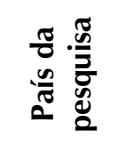 & $\begin{array}{l}\bar{\sigma} \\
\bar{a} \\
000 \\
\frac{0}{J}\end{array}$ & $\begin{array}{l}\frac{\widetilde{\sigma}}{5} \\
\frac{\pi}{\tilde{J}} \\
\text { S. }\end{array}$ & 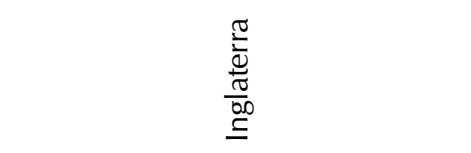 & $\begin{array}{l}\frac{0}{2} \\
\stackrel{\frac{\pi}{\pi}}{000} \\
\stackrel{0}{=}\end{array}$ \\
\hline 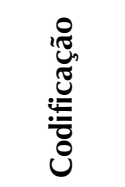 & 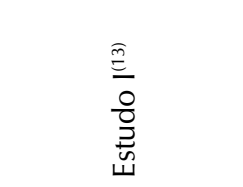 & 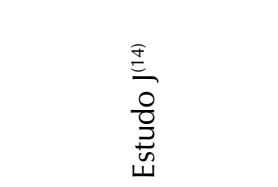 & 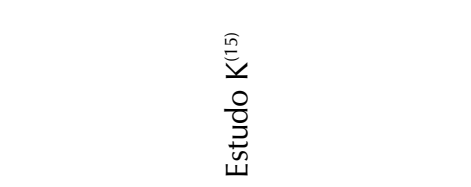 & 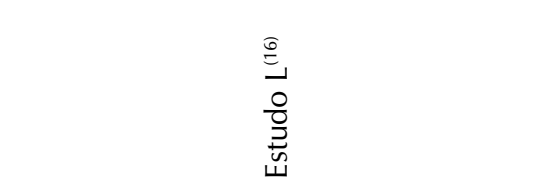 \\
\hline
\end{tabular}


Os estudos eram provenientes de diversos países da Europa (Inglaterra, Escócia e Espanha), Ásia (Japão, Índia e Irã) e Américas (Estados Unidos e Uruguai). Não foram encontrados estudos desenvolvidos no Brasil.

Em oito estudos, o curativo de hidrocolóide foi comparado a outros produtos, inclusive em dois estudos ocorreu comparação com dois produtos. A comparação mais freqüente foi entre hidrocolóide e curativo tradicional realizado com gaze (04). Em outros estudos foi comparado com malha de viscose pouco aderente (02). Destaca-se que um estudo avaliou o hidrocolóide com o curativo de acrílico transparente absorvente e outro a comparação ocorreu com o Biobrane ${ }^{\circledast}$. Nos dois estudos restantes sobre hidrocolóide não houve comparação por se tratar de pesquisa descritiva.

Quanto ao alginato de cálcio, esse foi comparado com a hidrofibra com prata em um estudo. No outro, a comparação ocorreu com gaze parafinada e filme semipermeável.

A amostra total dos estudos variou de 10 a 1.080 pacientes distribuídos em um ou dois ou três ou quatro grupos quando era estudo comparativo. Esses pacientes foram acompanhados em unidade de internação (08) ou ambulatorialmente (02). Em dois estudos esse dado não foi informado.

Dez estudos abordaram o uso da placa de hidrocolóide no tratamento de lesão cutânea. Destes, um estudo incluiu pacientes com lesões agudas e crônicas de diversas etiologias. Cinco estudos tiveram por amostra somente pacientes com lesões crônicas, sendo um estudo com de lesões de qualquer etiologia, dois sobre úlcera por pressão, dois sobre úlcera venosa. Os quatro estudos restantes contaram com pacientes com lesões agudas, com destaque para três estudos com ferida operatória (cirurgia abdominal, neurocirurgia e ferida operatória de cicatrização por segunda intenção) e um contou com pacientes com queimadura de pequena extensão.

Dois estudos analisaram a ação do alginato de cálcio no tratamento de lesão aguda (área doadora de pele) e crônica (pacientes com lesão em pé decorrente do diabetes melito).

Dos estudos obtidos, dois eram estudos de metanálise, sete eram estudos clínicos randomizados controlados, um era estudo controlado, mas sem randomização e dois eram descritivos, correspondendo ao nível de evidência II (07), nível III (01) e nível IV (02) Destaca-se que foram encontrados dois estudos com maior força de evidência, nível I, que eram os estudos de metanálise.

Foram identificados cinco desfechos analisados pelos estudos. Alguns avaliaram mais de um desfecho. $\mathrm{O}$ mais frequente foi "cicatrização" (09), seguido por "tempo de cura" (05). Os desfechos "taxa de cicatrização" e "infecção" foram avaliados por dois estudos o desfecho "redução da área lesada", por um.

Nos oito estudos comparativos sobre o hidrocolóide, este demonstrou superioridade em relação ao desfecho avaliado em três estudos. Apresentou desempenho inferior em um estudo, no qual foi comparado com curativo de acrílico transparente absorvente. Em quatro estudos não houve diferença estatística, mesmo quando o hidrocolóide foi comparado ao curativo tradicional.

Nos dois estudos referentes ao alginato de cálcio, esse produto demonstrou superioridade quando comparado com gaze parafinada e apresentou desempenho inferior quando comparado à hidrofibra.

A análise dos estudos permitiu estabelecer três recomendações para o uso da placa de hidrocolóide descritas no Quadro 4.

Quadro 4 - recomendações para a indicação da placa de hidrocolóide ou alginato de cálcio. Belo Horizonte-MG, 2013.

\begin{tabular}{|c|c|c|}
\hline $\begin{array}{c}\text { Recomendações para o uso da } \\
\text { placa de hidrocolóide ou alginato } \\
\text { de cálcio }\end{array}$ & $\begin{array}{l}\text { Nível de } \\
\text { evidência }\end{array}$ & Estudo \\
\hline \multirow{3}{*}{$\begin{array}{l}\text { 1- Usar cobertura de hidrocolóide } \\
\text { para tratar ferida operatória: } \\
\text { decorrente de cirurgia } \\
\text { abdominal ou neurocirurgia } \\
\text { ou cicatrização por segunda } \\
\text { intenção. }\end{array}$} & Nível I & Estudo $A^{(5)}$ \\
\hline & Nível II & Estudo $\mathrm{G}^{(11)}$ \\
\hline & Nível IV & Estudo I I \\
\hline \multirow{2}{*}{$\begin{array}{l}\text { 2- Usar cobertura de hidrocolóide } \\
\text { para tratar úlceras por pressão } \\
\text { de estágios II e III. }\end{array}$} & Nível I & Estudo $A^{(5)}$ \\
\hline & Nível II & Estudo $\mathrm{D}^{(8)}$ \\
\hline \multirow{2}{*}{$\begin{array}{l}\text { 3- Usar cobertura de hidrocolóide } \\
\text { associada à terapia compressiva } \\
\text { de multicomponentes de quatro } \\
\text { camadas para tratar úlcera } \\
\text { venosa. }\end{array}$} & Nível I & Estudo $A^{(5)}$ \\
\hline & Nível II & Estudo $C^{(7)}$ \\
\hline
\end{tabular}

\section{DISCUSSÃO}

Nos últimos 30 anos, os profissionais que cuidam de pessoas com lesão cutânea tiveram acesso a um arsenal de coberturas de tecnologia avançada. O conhecimento sobre a cicatrização de lesão progrediu significativamente durante as últimas décadas, como resultado de intensa investigação clínica e científica em torno da introdução e uso de coberturas. Hoje, o profissional compreende o conceito de meio úmido, cura, oclusão, custo-eficácia, preparação do leito lesão e atividade da metaloprotease, para citar alguns dos muitos conceitos no cuidado da lesão que surgiram em consequência do avanço da tecnologia.

O conceito de cicatrização de lesão em meio úmido iniciou na década de 1970 com a introdução de coberturas de filme de poliuretano e hidrocolóide, sendo que hoje em muitos países estes materiais são considerados coberturas tradicionais ${ }^{(17)}$. Esse fato foi confirmado nessa revisão, pois dos 12 artigos da amostra, o uso do hidrocolóide se deu em 10 estudos.

O hidrocolóide, originalmente foi utilizado no cuidado com estoma, sendo patenteado em 1967. Seu uso no tratamento de lesões cutâneas ocorreu nos anos de 1980. Os hidrocolóides 
clássicos consistem de polisorbutileno, carboximetilcelulose sódica, gelatina e pectina. As coberturas absorvem o fluido da lesão transformando-o em gel ${ }^{(18)}$. Os dados desse estudo revelam um maior interesse dos pesquisadores a respeito do uso da placa de hidrocolóide no tratamento de lesão aguda e crônica de diversas etiologias, que pode ser atribuído ao fato de que a mesma chegou ao mercado dos Estados Unidos e da Europa há mais de três décadas. No mercado brasileiro foi disponibilizado na década de 1990 e, o seu custo, então considerado elevado foi uma barreira inicial para sua difusão(19).

Revisão sistemática publicada em 1999 resultou em implicações para a prática confirmando ainda nessa época a existência de pouca evidência para indicar a cobertura ou agente tópico mais eficaz no tratamento de lesão crônica. No entanto, havia evidências de que as coberturas de hidrocolóide eram melhores do que coberturas secas para tratamento de úlceras por pressão. Porém, no tratamento de úlceras venosas, a cobertura de baixa aderência foi considerada tão eficaz quanto a de hidrocolóide sob bandagem de compressão ${ }^{(2)}$. Tais resultados foram semelhantes aos obtidos nessa revisão realizada em 2013, que contou com estudos publicados a partir de 2004, considerando que hidrocolóide foi superior quando usado no tratamento de úlcera por pressão. Mas, também no tratamento de úlceras venosas, o resultado foi o mesmo quando o hidrocolóide foi comparado com coberturas de baixa aderência associadas ao uso de terapia compressiva de longo estiramento ou de multicomponentes.

A placa de hidrocolóide tem sido geralmente recomendada no tratamento de úlcera por pressão de estágio II e III, com profundidade mínima ${ }^{(20)}$. Essa recomendação é sustentada por estudo de revisão sistemática ${ }^{(18)}$ que comprovou ser o hidrocolóide mais efetivo quando comparado com curativo de gaze na redução da área lesada. Tal achado é corroborado por metanálise publicada em 2005(21), a qual comprovou que o tratamento com placa de hidrocolóide aumenta significativamente a taxa de cicatrização de úlcera por pressão quando comparada com gaze umedecida em solução salina a 0,9\%. Entretanto, os autores afirmam que as evidências são insuficientes para se considerar uma cobertura mais efetiva do que a outra. Resultado diferente foi obtido em revisão sistemática sobre úlcera por pressão que recomenda o hidrocolóide no tratamento desse tipo de lesão amparada em evidências ${ }^{(22)}$.

Nessa revisão integrativa, quando a placa de hidrocolóide foi comparada com a cobertura transparente absorvente acrílica no tratamento dessas lesões, apresentou resultado inferior. A cobertura transparente absorvente acrílica faz parte da categoria de coberturas absorventes e apresenta características de cobertura impermeável e interativa. Interessante lembrar que a úlcera por pressão constitui um agravo passível de prevenção em detrimento da necessidade de tratamento ${ }^{(23)}$.

No tratamento de queimadura de espessura parcial há grande variedade de coberturas disponíveis no mercado. Melhorias na tecnologia e avanços no entendimento da cicatrização de lesões têm também tem impulsionado o desenvolvimento de novas coberturas para queimadura. Entretanto, revisão sistemática envolvendo 26 estudos clínicos randomizados controlados constatou que há uma escassez de estudos de alta qualidade metodológica sobre o uso de coberturas em queimaduras de espessura superficial e parcial. Os estudos analisados avaliaram uma variedade de intervenções. Apesar de alguns resultados potencialmente positivos, os autores afirmaram que grande parte advém de ensaios com deficiências metodológicas, sendo de pouca utilidade no auxílio dos profissionais no momento da escoIha de tratamento para queimadura ${ }^{(24)}$. Resultado semelhante também foi encontrado por essa revisão integrativa, pois o estudo sobre queimadura que compôs a amostra e no qual foi utilizado o hidrocolóide no tratamento de queimadura de espessura parcial não houve diferença significativa de tempo de cicatrização. Entretanto, estudo realizado ainda na década de $1990^{(25)}$, também com pacientes vítimas de queimaduras constatou que o hidrocolóide apresentou melhores resultados, inclusive na variável custo.

Algumas pesquisas têm sido realizadas para avaliar o desempenho do hidrocolóide no tratamento de feridas operatórias. Resultado de revisão sistemática sobre esse tema, com amostra de 13 estudos clínicos randomizados envolvendo ferida operatória por segunda intenção, evidenciou que a amostra consistia de pequenos ensaios, de má qualidade, tornando as provas insuficientes ${ }^{(25)}$. Contudo, os dois estudos sobre feridas operatórias decorrentes de cirurgias abdominais ou neurológicas dessa revisão integrativa apresentaram resultados distintos. Em um estudo não houve superioridade do hidrocolóide em relação às coberturas tradicionais de gaze. Mas, no outro estudo, a cicatrização decorrente do uso de hidrocolóide foi considerada "excelente". Outros autores ${ }^{(26)}$, discorrendo sobre tratamento de lesões cutâneas sem especificar a etiologia também enfatizam as qualidades do hidrocolóide, dentre elas: facilidade de aplicação da cobertura, conforto, diminuição da dor e não-exigência de trocas frequentes.

Apesar de não fazer parte do desfecho desta revisão integrativa, o aspecto econômico foi considerado nestes dois estudos. Os autores comprovaram ter menor custo o tratamento de ferida operatória com hidrocolóide, em detrimento das coberturas tradicionais envolvendo gaze e fita cirúrgica.

O alginato de cálcio foi avaliado por dois estudos dessa revisão integrativa. Essa cobertura é usada no tratamento de lesões cutâneas, sendo derivado de alga, biodegradável e pode ser encontrado na apresentação de cordão ou placa de consistência frouxa e tem sido aplicado com sucesso para limpar uma ampla variedade de lesões secretantes, com exsudato de moderado a intenso. É altamente absorvente, mantém um microambiente fisiologicamente úmido o que permite a troca gasosa e provê uma barreira para a contaminação, além de promover a cicatrização e a formação de tecido de granulação(27).

No estudo onde foram estudados pacientes com lesões em pés decorrentes de diabetes, os autores comprovaram melhores resultados com hidrofibra, recomendando-a preferencialmente para tratar lesões profundas com infecção. Porém, a ênfase neste trabalho foi em relação a tempo de cicatrização. No tocante à evolução de lesões em pés de pacientes diabéticos faz-se oportuno relembrar a gravidade dessa condição e a necessidade de prevenção dessas lesões, considerando-se que 
tais pacientes sofrem sérias mudanças na qualidade de vida, ocasionadas pela diminuição da capacidade de locomoção, tornando-os dependentes de seus familiares ${ }^{(28)}$.

Fez parte dessa revisão integrativa um estudo com pacientes submetidos a enxertos de pele, cujas áreas doadoras foram tratadas com uma dentre quatro opções, incluindo o alginato de cálcio. Foram obtidos melhores resultados nos pacientes tratados com alginato de cálcio associados a filme semipermeável. Estudo ${ }^{(29)}$ sobre o mesmo tema realizado com amostra de 57 pacientes submetidos a enxertos de pele também considerou o alginato de cálcio como melhor cobertura para esse tipo de lesão. Esses locais foram tratados com coberturas de alginato de cálcio, porém com cobertura secundária tradicional. Todas as lesões reepitelizaram sem complicações em período máximo de 10 dias. Os autores também constataram a propriedade de absorção do alginato de cálcio, que eliminou o problema da formação de seroma e perdas de exsudato visto rotineiramente com o uso de filme de poliuretano.

\section{CONSIDERAÇÕES FINAIS}

A análise dos resultados desse estudo permitiu estabelecer três recomendações sobre o uso apenas de cobertura de hidrocolóide no tratamento de lesão cutânea crônica. Esses dados irão subsidiar a prática clinica dos profissionais responsáveis pela indicação de cobertura.

A diversidade de desfechos analisados pelos estudos da amostra impossibilitou a realização de metanálise, sendo, portanto, utilizada análise descritiva dos resultados.

Recomenda-se que sejam realizados outros estudos clínicos randomizados controlados, com amostra calculada previamente e com análise de desfecho único. Estudos devem relatar resultado como cura completa da lesão, que é mais importante do ponto de vista do paciente do que a percentagem de redução na área da lesão ou tempo de cura para facilitar a realização futura de metanálise, a fim de elucidar questões ainda obscuras.

\section{REFERÊNCIAS}

1. Seaman S. Dressing selection in chronic wound management. J Am Podiatr Med Assoc 2002;92(1):24-33.

2. Bradley $M$, Cullum N, Nelson EA, Petticrew M, Sheldon T, Torgerson D. Systematic reviews of wound care management: (2) dressings and topical agents used in the healing of chronic wounds. Health Technol Assess 1999;3(17):1-35.

3. Whittemore $R$, Knafl K. The integrative review: updated methodology. J Adv Nurs 2005;52(5):546-53.

4. Steler CB, Morsi D, Rucki S, Broughton S, Corrigan B, Fitzgerald J, et al. Utilization-focused integrative reviews in a nursing service. Appl Nurs Res 1998;11(4):195-206.

5. Singh A, Halder S, Menon GR, Chumber S, Misra MC, Sharma LK, et al. Meta-analysis of randomized controlled trials on hydrocolloid occlusive dressing versus conventional gauze dressing in the healing of chronic wounds. Asian J Surg 2004;27(4):326-32.

6. Palfreyman S, Nelson A, Jonathan A, Michaels JA. Dressings for venous leg ulcers: systematic review and metaanalysis. BMJ [periódico na internet]. 2007 [acesso em 01 out 2010] Disponível em www.bmj.com/content/335/7613/244.abstract.

7. Nelson EA, Prescott RJ, Harper DR, Gibson B, Brown D, Ruckley CV. A factorial, randomized trial of pentoxifylline or placebo, four-layer or single-layer compression, and knitted viscose or hydrocolloid dressings for venous ulcers. J Vasc Surg 2007;45(1):134-41.

8. Hollisaz MT, Khedmat H, Yari F. A randomized clinical trial comparing hydrocolloid, phenytoin and simple dressings for the treatment of pressure ulcers. BMC Dermatol 2004;4(1): 18.

9. Brown-Etris M, Milne C, Orsted H, Gates JL, Netsch D, Punchello $M$, et al. A prospective, randomized, multisite clinical evaluation of a transparent absorbent acrylic dressing and a hydrocolloid dressing in the management of Stage II and shallow Stage III pressure ulcers. Adv Skin Wound Care 2008;21(4):169-74.

10. Cassidy C, Peter SD, Lacey S, Beery M, Ward-Smith P, Sharp RJ, et al. Biobrane versus duoderm for the treatment of intermediate thickness burns in children: a prospective, randomized trial. Burns 2005;31(7):890-3.

11. Shinohara T, Yamashita $Y$, Satoh $K$, Mikami K, Yamauchi $Y$, Hoshino $S$, et al. Prospective evaluation of occlusive hydrocolloid dressing versus conventional gauze dressing regarding the healing effect after abdominal operations: Randomized controlled trial. Asian J Surg 2008;31(1):1-5.

12. Fujimoto $Y$, Shimooka N, Ohmishi Y, Yoshimine T. Clinical evaluation of hydrocolloid dressings for neurosurgical wounds. Surg Neurol 2008;70(2):217-220.

13. Bazzano C, Alvarez M, Martínez M. Apósitos hidrocoloidales en la cicatrización por segunda intención de heridas quirúrgicas. Rev Méd Urug 2008;24(1):32-6.

14. Aparicio EG, Castilla PC, Díez GMT, Dorado A, Mendoza G, Núñez FJM, et al. Therapeutic behavior of a hydrocolloid dressing. Its evolution in the treatment of acute and chronic dermal ulcers. Rev Enferm 2005;28(12):49-55.

15. Jude EB, Apelqvist J, Spraul M, Martini J. Prospective randomized controlled study of Hydrofiber dressing containing ionic silver or calcium alginate dressings in non-ischaemic diabetic foot ulcers. Diabet Med 2007;24(3):280-8.

16. Beldon P. Comparison of four different dressings on donor site wounds. Br J Nurs 2004;13(6 suppl.):38-45.

17. Queen D, Orsted H, Sanada H, Sussman G. A dressing history. Int Wound J 2004;1(1):59-77.

18. Heyneman A, Beele H, Vanderwee K, Defloor T. A systematic review of the use of hydrocolloids in the treatment of pressure ulcers. J Clin Nurs 2008;17(9):1164-73. 
19. Mandelbaum SF, Di Santis EP, Mandelbaum MHS. Cicatrização: conceitos atuais e recursos auxiliares - Parte I. An Bras Dermatol 2003;78(4):393-410.

20. National Pressure Ulcer Advisory Panel, European Pressure Ulcer Advisory Panel. In: Prevention and treatment of pressure ulcers: clinical Practice Guideline. Washington.: National Pressure Ulcer Advisory Panel; 2009. P. 51-120.

21. Bouza C, Saz Z, Muñoz A, Amate JM. Efficacy of advanced dressings in the treatment of pressure ulcers: a systematic review. J Wound Care 2005;14(5):193-9.

22. Rocha JA, Miranda MJ, Andrade MJ. Abordagem terapêutica das úlceras por pressão - intervenções baseadas em evidências. Acta Med Port 2006;19:29-38.

23. Louro M, Ferreira M, Povoa P. Avaliação de protocolo de prevenção e tratamento de úlceras de pressão. RBTI 2007;19(3):337-41.

24. Wasiak J, Cleland H, Campbell F. Dressings for superficial and partial thickness burns. Cochrane Database Syst Rev 2008:(4) .

25. Wyatt D, McGowan DN. Comparison of hydrocolloid dressing and silver sulfadiazine cream in the outpatient management of second degree burns. J Trauma 1990;30(7):857-65.

26. Vermeulen H, Ubbink D T, Goossen A, Vos R, Legemate DA. Systematic review of dressings and topical agents for surgical wounds healing by secondary intention. Br J Surg 2005;92(6):665-72

27. Jones V, Grey JE, Harding KG. Wound dressings. BMJ 2006;332(7544):777-780.

28. Lee KY, Mooney DJ. Alginate: properties and biomedical applications. Prog Polym Sci 2012;37(1):106-126.

29. Prog Polym Sci. 2012; 37(1): 106-126

30. Jorge $\mathrm{BH}$ et al. Análise clínica e evolução de 70 casos de lesões podais infectadas em pacientes diabéticos. Arq Bras Endocrinol Metab 1999;43(5):366-72. 